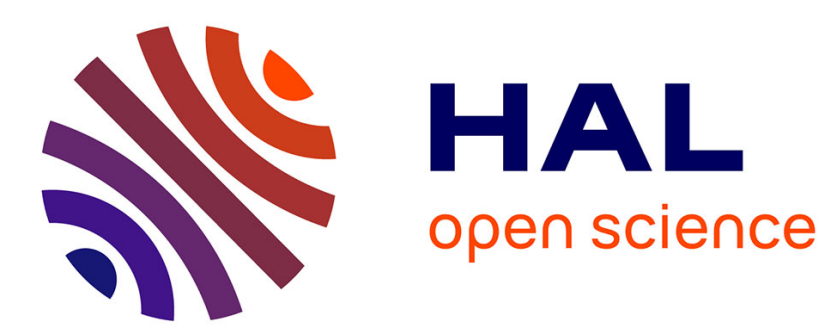

\title{
La polyembryonie haploïdediploïde chez le lin (Linum usitatissimum L.). Etude cytologique et physiologique Christian Huyghe
}

\section{To cite this version:}

Christian Huyghe. La polyembryonie haploïdediploïde chez le lin (Linum usitatissimum L.). Etude cytologique et physiologique. Agronomie, 1987, 7 (8), pp.567-573. hal-00885028

\section{HAL Id: hal-00885028 \\ https://hal.science/hal-00885028}

Submitted on 1 Jan 1987

HAL is a multi-disciplinary open access archive for the deposit and dissemination of scientific research documents, whether they are published or not. The documents may come from teaching and research institutions in France or abroad, or from public or private research centers.
L'archive ouverte pluridisciplinaire HAL, est destinée au dépôt et à la diffusion de documents scientifiques de niveau recherche, publiés ou non, émanant des établissements d'enseignement et de recherche français ou étrangers, des laboratoires publics ou privés. 


\title{
La polyembryonie haploïde-diploïde chez le lin (Linum usitatissimum L.). Etude cytologique et physiologique
}

\author{
Christian HUYGHE
}

I.N.R.A., Station de Génétique et d'Amélioration des Plantes. Centre de Recherches de Versailles, Route de Saint-Cyr, F 78000 Versailles $\left({ }^{I}\right)$

\begin{abstract}
RÉSUMÉ
L'origine de la polyembryonie haploïde-diploïde chez les génotypes de lin (Linum usitatissimum L.) « Rocket 4 » et « RA91 » réside dans la division avant fécondation de l'oosphère en deux oosphères indépendantes dont l'une sera fécondée et l'autre se développera parthénogénétiquement en embryon haploïde. Ce phénomène, favorisé par la culture des plantes à hautes températures, s'amplifie également avec l'âge du gamétophyte au moment de la fécondation.
\end{abstract}

Mots clés additionnels : Ovule, sac embryonnaire, division de l'oosphère. L.).

The origin of haploid-diploid twins in flax (Linum usitatissimum L.) genotypes 'Rocket 4' and 'RA91' was found to be the division of the egg cell to give two independent egg cells, one which is fertilized and the other of which develops parthenogenetically into a haploid embryo. The twinning frequency increased with high temperatures during plant growth and flowering and with age of the macrogametophyte at fertilization.

Additional key words : Ovule, embryo sac, egg cell mitosis.

\section{INTRODUCTION}

Dès 1933, KAPPERT signalait l'existence de polyembryons haploïdes-diploïdes chez le lin (Linum usitatissimum L.). Un certain nombre de génotypes présentent, avec des taux faibles, des polyembryons dont 85 p. 100 sont des couples n-2n (RAJHATHY, 1976). Cependant, au sein de la variété oléagineuse canadienne « Rocket », il a été possible de sélectionner le génotype «Rocket 4 » à niveau élevé de polyembryonie. A partir de cette lignée, PLESSERS (1965) observait, après 7 générations de sélection, un taux de 2,9 p. 100 ; RAJHATHY (1976) parmi 49 lignées haploïdes doublées obtenait une variation de 0,1 p. 100 à 8,7 p. 100 pour une moyenne de 4 p. 100 et MURRAY (1980) des plantes avec des taux de 15,5 p. 100.

En 1983, GREEN \& SALISBURY, en croisant ce génotype avec la variété oléagineuse «Avantgarde »

(1) Adresse actuelle : I.N.R.A., Station d'Amélioration des Plantes fourragères, F 86600 Lusignan. très faiblement polyembryogène ont obtenu une transgression en F2 avec une plante donnant 32 p. 100 de polyembryonie.

L'origine cellulaire des polyembryons n'est pas connue avec précision. KAPPERT (1933) proposait le développement d'une synergie simultanément au développement du zygote issu de la fécondation. Plus récemment, THOMPSON (1977) montrait aussi l'origine strictement femelle de l'embryon haploïde, l'embryon diploïde ayant une origine hybride. En 1985, MURRAY sur la base d'observations génétiques de descendances de croisements entre lignées polyembryogènes et lignées non polyembryogènes supposait le développement de 2 mégaspores.

Aucun examen cytologique n'a été effectué qui permettrait de trancher entre ces différentes hypothèses relatives à l'origine des polyembryons haploïdesdiploïdes chez le lin. Les objectifs de cette étude sont d'une part de rechercher à l'aide d'une technique cytologique rapide, l'origine cellulaire des polyembryons et, d'autre part, de préciser l'influence du milieu sur 
l'expression du phénomène ainsi que son évolution avec l'âge du gamétophyte femelle.

\section{MATÉRIELS ET MÉTHODES}

« Rocket 4 » est un génotype oléagineux à fleurs bleues issu du cultivar canadien « Rocket ". Il présente en serre un taux de polyembryonie moyen de 4 p. 100 mais avec des variations importantes, de $0,5 \mathrm{p} .100$ à 11,2 p. 100 (HUYGHE, 1985). Les plantes que nous avons utilisées sont issues de deux générations d'autofécondation.

RA91 est issu du croisement «Rocket 4 » $\times$ " Avantgarde ». La descendance d'une plante F3 issue de la plante $\mathrm{F} 2$ à $32 \mathrm{p} .100$ de polyembryons a été reçue de GreEn et SAlisbury. Pour les études cytologiques et l'étude de l'influence de la température, nous avons utilisé une lignée haploïde doublée RA91 HD8 à fleurs bleues obtenue parmi la descendance de la plante $F 3$. L'haploïde, jumeau d'une plante diploïde, est traité à la colchicine. Pour cela, au début de la floraison, la tige est coupée sous l'inflorescence et l'on renverse sur la section un petit tube contenant du coton hydrophile inbibé d'une solution aqueuse de colchicine à la concentration de $2 \cdot 10^{-4} \mathrm{~g} / \mathrm{ml}$ additionné de Tween 80 . Le dispositif est laissé en place durant $20 \mathrm{~h}$. La partie supérieure de la tige se nécrose sur quelques centimètres. En dessous, les premiers bourgeons qui se développent ont généralement doublé leur stock chromosomique et produisent après floraison des graines haploïdes doublées. Le temps nécessaire entre le traitement et l'obtention de graines mûres est de 9 à 11 semaines, le nombre de graines variant de 1 à 100 .

L'ensemble des plantes est cultivé en pots de $12 \mathrm{~cm}$ de diamètre. Pour l'étude de l'influence de la température, les différentes conditions thermiques sont $16^{\circ} \mathrm{C}$ de jour et $12{ }^{\circ} \mathrm{C}$ de nuit $\left(16^{\circ} \mathrm{C} / 12^{\circ} \mathrm{C} \mathrm{J} / \mathrm{N}\right)$, $24{ }^{\circ} \mathrm{C} / 18{ }^{\circ} \mathrm{C} \mathrm{J} / \mathrm{N}, 24{ }^{\circ} \mathrm{C} / 12{ }^{\circ} \mathrm{C} \mathrm{J} / \mathrm{N}$ sous une photopériode de $16 \mathrm{~h}$ et une intensité lumineuse de 7000 lux au niveau des plantes. Lors de l'analyse cytologique de l'influence de l'âge de l'ovule au moment de la fécondation, les plantes du génotype "Rocket 4 " sont cultivées sous une température constante de $24^{\circ} \mathrm{C}$ avec la même intensité lumineuse et la même photopériode. Les fleurs sont alors castrées la veille de l'anthèse $(\mathrm{J}-1)$. De $\mathrm{J}-1$ à $\mathrm{J}+2,2$ séries de prélèvements sont effectuées, la première avec 4 prélèvements quotidiens $(-2 \mathrm{~h},+5 \mathrm{~h},+10 \mathrm{~h},+15 \mathrm{~h}$ par rapport à l'allumage des lampes), la seconde avec 2 prélèvements quotidiens $(+5 \mathrm{~h},+15 \mathrm{~h}$ par rapport à l'allumage des lampes). Chaque prélèvement comprend 7 capsules soit 70 ovules.

Les études cytologiques ont été réalisées à l'aide d'une technique par éclaircissage (LEvIEIL \& HUYGHE, 1985) issue d'une modification de la technique de HERR (1971). Le protocole est le suivant :

- dissection des ovules,

- 3 h de fixation dans le FPA50 (BLACKBURN \& CHRISTOPHEL, 1976) : $90 \mathrm{ml}$ d'éthanol $50,7 \mathrm{ml}$ de formaldéhyde $40,3 \mathrm{ml}$ d'acide propionique pur,

- $30 \mathrm{mn}$ dans l'acide lactique 85 p. 100 pour un pré-éclaircissage,

- $1 \mathrm{~h}$ dans le liquide de Herr composé de phénol, d'hydrate de chloral, d'acide lactique 85 p. 100 , d'essence de girofle et de xylène dans les proportions 22-2-2-1 en poids. Le montage se fait dans une goutte de mélange éclaircissant entre lame et lamelles. Sur chaque lame, 2 lamelles, espacées de $1 \mathrm{~cm}$, ont été préalablement collées afin d'éviter l'écrasement quand la troisième lamelle est posée sur l'ensemble. L'observation se fait en microscopie à contraste de phase soit de Zernicke soit interférentiel de Nomarski.

\section{RÉSULTATS}

\section{A. Etude qualitative}

La polyembryonie consiste en la présence, dans une graine, de 2 ou plusieurs embryons, ces embryons étant inclus dans une même membrane blanchâtre constituée par les restes de l'albumen. Les 2 embryons sont issus d'un même sac embryonnaire.

Le sac embryonnaire est une cavité renflée du côté micropylaire qui se rétrécit vers la chalaze. Il est entouré d'une épaisse couche de cellules allongées.

En observant par transparence des ovules de génotypes non polyembryogènes avant pollinisation, on distingue les différentes cellules qui composent le sac. A l'extrémité chalazienne, se trouvent 3 ou 4 antipodes. Au moment de l'anthèse, leurs noyaux sont petits, inclus dans une masse de cytoplasme, avec un contour régulier et un nucléole dense. A l'extrémité micropylaire, on reconnait les autres cellules du sac (fig. 1). Largement engagées dans le rétrécissement du micropyle, les synergides possèdent au moment de l'anthèse des noyaux petits, au contour irrégulier et avec un nucléole dense. Leurs vacuoles sont visibles en arrière du noyau. Appliquée contre la paroi des synergides, l'oosphère présente un noyau parfaitement sphérique avec un nucléole fréquemment creusé d'une vacuole centrale. Situés en arrière de l'oosphère, les noyaux polaires fusionnent $30 \mathrm{~h}$ environ avant l'anthèse. Au moment de l'anthèse, le noyau secondaire occupe une position centrale. De forme oblongue, il présente généralement un gros nucléole sphérique avec plusieurs vacuoles. La cellule de l'initiale de l'albumen a un cytoplasme très vacuolisé qui occupe le reste du sac embryonnaire.

Le type pollinique atteint le sac $7 \mathrm{~h}$ après la pollinisation pour une température de $22^{\circ} \mathrm{C}$ environ. Progressant à travers une des synergides qui dégénère, il s'arrête de croître à hauteur de l'oosphère et l'extrémité s'ouvre. Le premier noyau mâle s'accole au noyau secondaire. Il y a alors caryogamie et constitution du premier noyau de l'albumen (fig. 2). Une heure plus tard, le second noyau mâle vient s'accoler à l'oosphère pour donner, après fusion, le premier noyau zygotique.

L'étude d'ovules des génotypes polyembryogènes, " Rocket 4 » et « RA91 », en absence de pollinisation permet de comprendre l'origine des polyembryons. Si l'on retrouve facilement les 2 synergides, les noyaux polaires fusionnés et les 3 ou 4 antipodes, on observe dans un certain nombre d'ovules au moment de l'anthèse la présence de 2 noyaux de type " oosphère ", au contour régulier avec un nucléole gros et parfois creusé d'une vacuole (fig. 3). L'origine de ces 2 oosphères réside dans la division de l'oosphère 


\section{Figure 1}

Extrémité micropylaire du sac embryonnaire, avec les 2 synergies (s), l'oosphère (o) et les noyaux polaires fusionnés (np) $(\times 300)$.

Micropylar end of the embryo sac with the two synergids (s), the egg cell (o), and the fused polar nuclei $(n p)(\times 300)$.

Figure 2

Fécondation des noyaux polaires $(\times 1500)$.

Fertilization of the polar nuclei $(\times 1500)$.
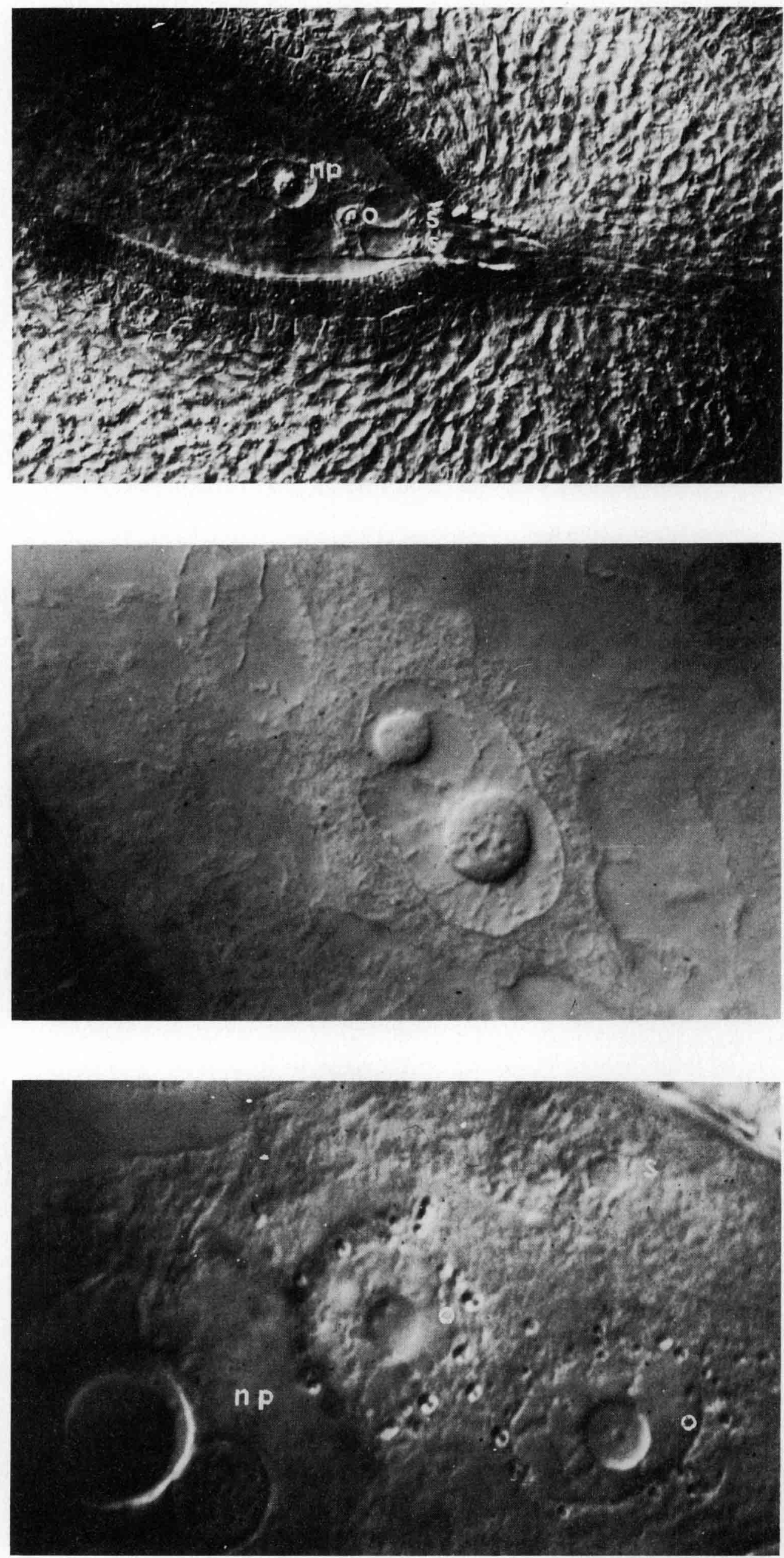

Figure 3

Sac embryonnaire d'un génotype polyembryogène avant fécondation. Extrémité micropylaire. Outre les noyaux polaires fusionnés (np) et une synergide (s), on reconnaît 2 noyaux de type 《oosphère » (o) $(\times 1500)$.

Micropylar end of an embryo sac of a polyembryogenic genotype before fertilization. In addition to the fused polar nuclei (np) and a synergid (s), the two egg cell type nuclei (o) are also present $(\times 1500)$ 

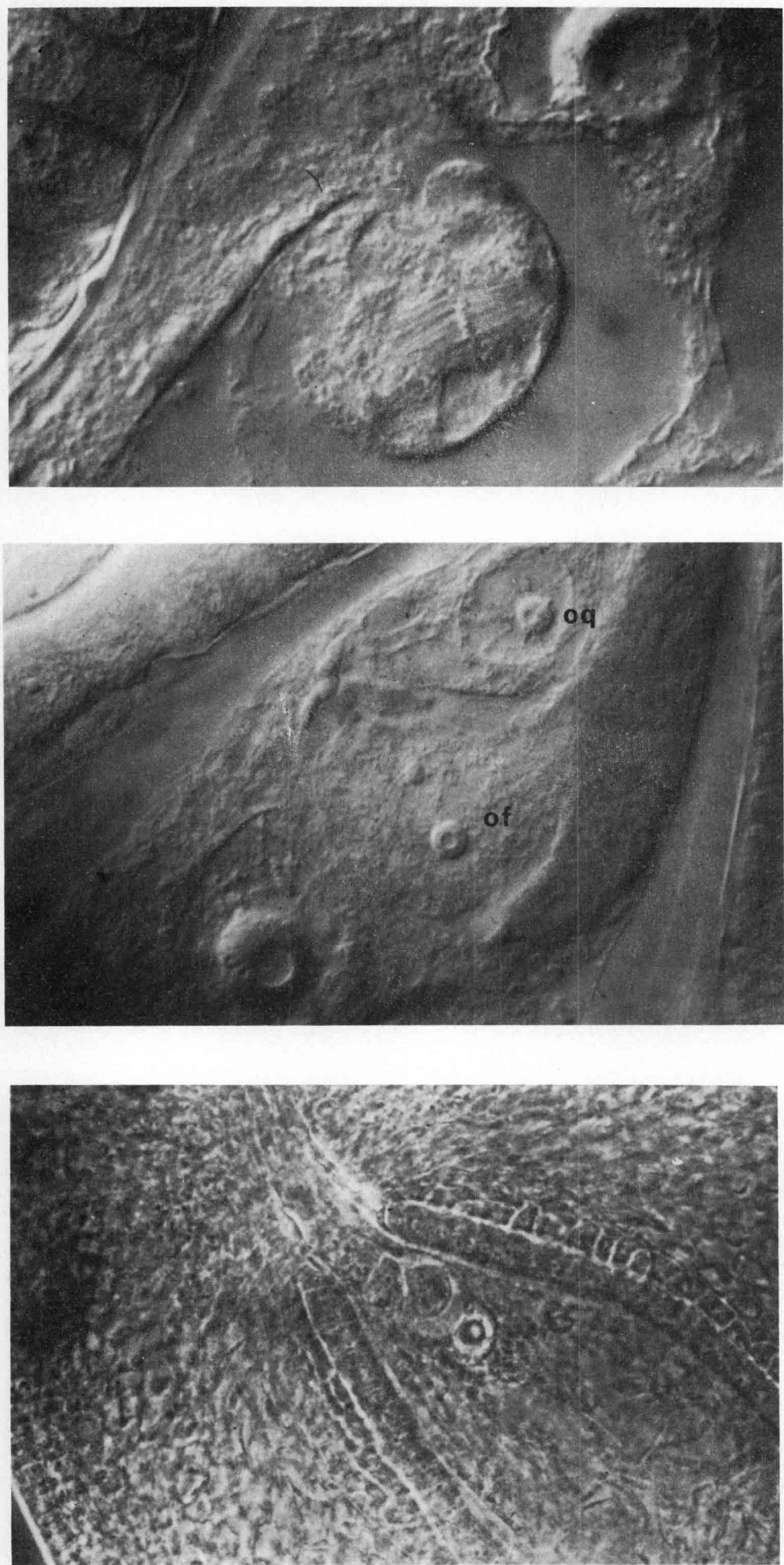

Figure 4

Fuseau mitotique de la division de l'oosphère avant fécondation ( $\times 1500)$.

Mitotic spindle of egg cell mitosis before fertilization $(\times 1500)$.

Figure 5

Fécondation en présence de 2 oosphères. Une oosphère est fécondée (of), tandis que l'autre reste quiescente (oq) $(\times 1500)$.

Ferilization when two egg cells are present. One is fertilized (of), the other remains unfertilized (oq) $(\times I 500)$

Figure 6

Embryon haploïde structuré dans le micropyle avant fécondation $(\times 700)$.

$A$ haploid embryo in the micropyle before fertilization $(\times 700)$. 
initiale, division mise en évidence par la présence d'un fuseau mitotique (fig. 4). Il se forme ainsi 2 cellules indépendantes et équipotentielles.

La chronologie de la double fécondation est similaire à celle observée chez les génotypes non polyembryogènes. Le premier noyau spermatique va fusionner avec le noyau secondaire pour constituer le premier noyau de l'albumen, le second s'accolant apparemment au hasard à une des 2 oosphères. Durant la fécondation d'une des oosphères, l'autre reste quiescente (fig. 5). La fécondation d'une oosphère va permettre la formation de l'embryon diploïde, l'autre oosphère pouvant se développer parthénogénétiquement en embryon haploïde.

Ce mécanisme peut présenter quelques déviations.

- Dans quelques rares cas, l'activité mitotique des oosphères peut se poursuivre donnant naissance, après division d'une des oosphères, à une structure à 3 oosphères indépendantes. Cela permet d'expliquer les cas de polyembryonie haploïde-haploïde-diploïde après fécondation d'une oosphère et développement parthénogénétique des 2 autres.

- Parfois, les 2 cellules issues de la première division ne s'individualisent pas, continuent à se diviser et donnent naissance à un proembryon haploïde (fig. 6). Le suspenseur, profondément enfoncé dans le micropyle, empêche l'entrée du tube pollinique ce qui condamne la survie de l'embryon haploïde faute d'albumen.

- L'étude d'ovules d'hybrides F1 entre RA91 et la variété non polyembryogène "Crocus » a mis en évidence dans le sac embryonnaire de quelques-uns d'entre eux un grand nombre de noyaux de type "noyaux polaires » ainsi que des fuseaux mitotiques issus des noyaux polaires. Ces proliférations semblent commencer avant la fin de la maturation du sac.

\section{B. Etude quantitative}

L'expression de la polyembryonie paraît très sensible aux conditions de milieu. Nous avons cherché à déterminer l'influence des conditions thermiques et l'évolution du phénomène en fonction de l'âge de l'ovule au moment de la fécondation.

Nous avons étudié l'influence des conditions thermiques sur le taux de division des oosphères $8 \mathrm{~h}$ après anthèse en absence de pollinisation et sur le taux de polyembryonie. Les taux de polyembryons estimés sur 10 plantes de RA91 HD8 dans chacune des conditions thermiques (tabl. 1) montrent l'influence défavorable des températures basses, $16{ }^{\circ} \mathrm{C} / 12{ }^{\circ} \mathrm{C} \mathrm{J} / \mathrm{N}$, avec un taux moyen de 2 p. 100 alors que ceux observés pour

TABLE 1

Effet des conditions de température sur le taux de polyembryonie observé sur la lignée RA91 HD8.

Effect of temperature conditions on the twinning rate of genotype RA9I HD8.

\begin{tabular}{ccc}
\hline \hline $\begin{array}{c}\text { Conditions } \\
\text { thermiques }\end{array}$ & $\begin{array}{c}\text { Taux moyen de } \\
\text { polyembryonie (\%) }\end{array}$ & $\begin{array}{c}\text { Valeurs extrêmes } \\
(\%)\end{array}$ \\
\hline $16^{\circ} \mathrm{C} / 12{ }^{\circ} \mathrm{C}$ & 2,0 & $1-3$ \\
$24^{\circ} \mathrm{C} / 18{ }^{\circ} \mathrm{C}$ & 27,9 & $14-44$ \\
$28^{\circ} \mathrm{C} / 12^{\circ} \mathrm{C}$ & 26,8 & $20-47$ \\
\hline \hline
\end{tabular}

$24{ }^{\circ} \mathrm{C} / 18{ }^{\circ} \mathrm{C} \mathrm{J} / \mathrm{N}$ et $28{ }^{\circ} \mathrm{C} / 12{ }^{\circ} \mathrm{C} \mathrm{J} / \mathrm{N}$ sont beaucoup plus élevés ( 27,9 p. 100 et 26,8 p. 100 respectivement).

Les taux de division des oosphères $8 \mathrm{~h}$ après anthèse en l'absence de pollinisation reflètent la situation au moment estimé de la fécondation de l'oosphère. Ces taux mesurés sur 6 plantes, à raison de 50 ovules par plante, dans chacune des conditions thermiques (tabl. 2) sont très élevés à $24{ }^{\circ} \mathrm{C} / 18{ }^{\circ} \mathrm{C}(60 \mathrm{p} .100)$ et à $28^{\circ} \mathrm{C} / 12^{\circ} \mathrm{C}(52 \mathrm{p}$. 100) mais non négligeables à $16{ }^{\circ} \mathrm{C} / 12{ }^{\circ} \mathrm{C}(34$ p. 100). Les températures élevées ont donc un effet favorable à la fois sur la division de l'oosphère et sur le développement des polyembryons obtenus après fécondation.

TABLE 2

Effet des conditions de température sur le taux d'oosphères divisées, $8 \mathrm{~h}$ après anthèse, en l'absence de pollinisation sur RA91 HD8.

Effect of temperature conditions on the rate of divided egg cells, $8 \mathrm{~h}$ after anthesis, without pollination, in genotype RA91 HD8.

\begin{tabular}{ccc}
\hline \hline $\begin{array}{l}\text { Conditions } \\
\text { thermiques }\end{array}$ & $\begin{array}{l}\text { Taux moyen } \\
\text { d'oosphères } \\
\text { divisées }(\%)\end{array}$ & $\begin{array}{c}\text { Valeurs extrêmes } \\
(\%)\end{array}$ \\
\hline $16^{\circ} \mathrm{C} / 12^{\circ} \mathrm{C}$ & 31 & $8-52$ \\
$24^{\circ} \mathrm{C} / 18^{\circ} \mathrm{C}$ & 60 & $51-74$ \\
$28^{\circ} \mathrm{C} / 12^{\circ} \mathrm{C}$ & 52 & $30-74$ \\
\hline
\end{tabular}

La mesure de la largeur des ovules prélevés $8 \mathrm{~h}$ après anthèse à $24^{\circ} \mathrm{C} / 18{ }^{\circ} \mathrm{C}$ et $28{ }^{\circ} \mathrm{C} / 12^{\circ} \mathrm{C}$ montre une faible variation intra-capsule pour ce caractère mais il apparaît une corrélation positive entre le nombre d'ovules d'une capsule avec division de l'oosphère et la largeur moyenne des ovules de cette capsule : 0,670 à $24{ }^{\circ} \mathrm{C} / 18{ }^{\circ} \mathrm{C}$ et 0,650 à $28^{\circ} \mathrm{C} / 12^{\circ} \mathrm{C}$.

L'évolution des taux de division des oosphères selon l'âge de l'ovule, en absence de pollinisation, a été suivie chez « Rocket 4 » par l'étude de 3 caractères : 1) le taux de mortalité estimé par le pourcentage d'ovules où les noyaux sont en cours de dégénérescence, 2) le taux d'ovules avec oosphère divisée pour donner soit 2 ou plusieurs oosphères soit un embryon haploïde structuré, 3) le taux d'embryons haploïdes structurés.

Les résultats présentés figure 7 montrent que :

a) le taux de mortalité reste inférieur à 6 p. 100 jusqu'à la fin de $\mathrm{J}+1$ où il augmente brutalement à plus de 30 p. 100 . A la température constante de $24^{\circ} \mathrm{C}$, c'est à ce moment que surviennent une détérioration brutale des structures du sac embryonnaire et une diminution de la turgescence des stigmates. Cette augmentation du taux de mortalité signifie la fin de la possibilité de pollinisation et de la réussite de la fécondation.

$b$ ) le pourcentage d'ovules vivants présentant une division de l'oosphère manifeste une progression constante depuis 25 p. 100 au moment théorique de l'anthèse jusqu'à 72 p. 100 à la fin de $J+1$.

c) la courbe figurant le taux d'ovules vivants avec embryon haploïde structuré ne présente pas d'accroissement important. L'augmentation du taux de division révèle une augmentation du nombre des cas avec 2 ou plusieurs oosphères indépendantes, cas à l'origine des polyembryons.

Cette évolution favorable des taux de division est 


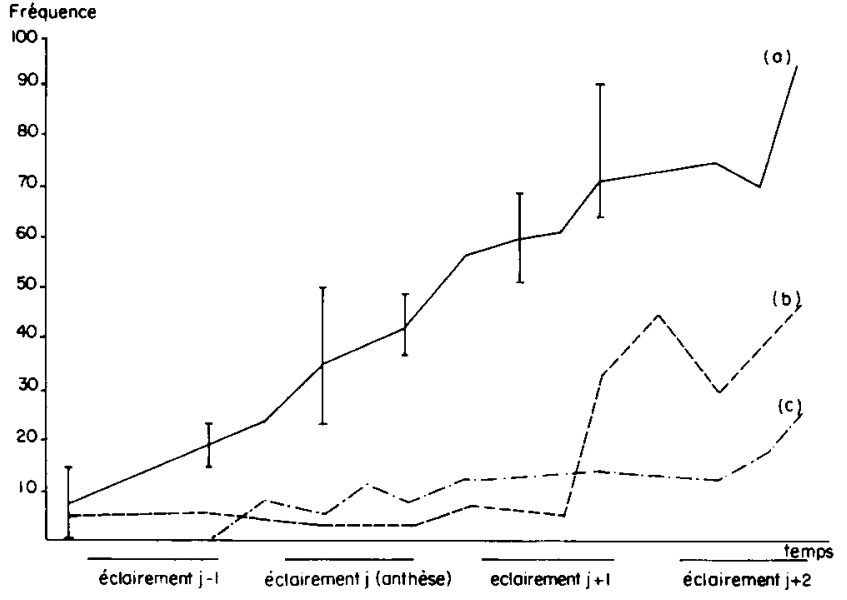

Figure 7

Evolution à $24^{\circ} \mathrm{C}$ de fleurs castrées de "Rocket 4 》.

a) ovules vivants avec oosphere divisee,

b) taux de mortalité,

c) ovules vivants avec un embryon haploide.

Development at $24^{\circ} \mathrm{C}$ of emasculated flowers of genotype " Rocket 4 m.

a) viable ovules with divided egg cell,

b) mortality rate.

c) viable ovules with a haploid embryo.

confirmée par l'augmentation des taux de polyembryonie suite à des pollinisations à différents moments sur des fleurs castrées la veille de l'anthèse. Le taux de polyembryonie sur «Rocket 4 » progresse ainsi de $0,75 \mathrm{p} .100$ pour une pollinisation la veille de l'anthèse normale à 2,2 p. 100 le jour de l'anthèse et à 5,5 p. 100 le lendemain.

\section{DISCUSSION}

Selon ces observations, la division avant fécondation de l'oosphère en 2 oosphères indépendantes apparaît comme une nouvelle explication de l'origine des polyembryons. Cette hypothèse a également été avancée pour les cas de polyembryonie observés sur des lignées de restauration de maïs après un long retard de pollinisation (ZVERZHANSKAYA \& GRISHINA, 1980). Cette nouvelle hypothèse vient s'ajouter aux différentes théories explicatives de la polyembryonie : présence de plusieurs sacs embryonnaires chez le riz (RAMIAH et al., 1935), l'asperge (RANDALL \& RICK, 1945) ; développement de 2 ou plusieurs mégaspores chez le soja (KENNEL \& HORNER, 1985) ; développement parthénogénétique d'une synergie simultanément au développement du zygote issu de la fécondation de l'oosphère chez Nicotiana, Lilium (COOPER, 1943), et Capsicum (DUMAS DE VAulX, 1977).

Chez le lin, MURRAY (1985) a apporté la preuve génétique que plusieurs mégaspores peuvent être à l'origine d'un couple de polyembryons haploïdesdiploïdes obtenu à partir de structures hybrides. Ce phénomène à faible fréquence peut être rapproché des observations de nombreux noyaux dans le sac embryonnaire d'ovules d'hybrides F1. Outre l'origine principale des polyembryons qu'est la division de l'oosphère avant fécondation, d'autres déviations du déroulement normal de la mégasporogenèse et de la mégagamétogenèse peuvent donc conduire à l'obtention de jumeaux haploïde-diploïdes.

La formation de polyembryons étant physiologiquement liée à la division de l'oosphère avant fécondation, l'observation cytologique d'ovules non fécondés constitue un moyen efficace de repérage précoce des génotypes polyembryogènes, ce repérage pouvant s'effectuer sur les premiers boutons floraux de chaque plante.

L'observation des taux de division et la mesure simultanée de la largeur des ovules a permis de mettre en évidence une corrélation inter-capsule positive entre ces 2 caractères simultanément à une corrélation intracapsule faible. Cet effet capsule signifie que le phénomène de division évolue avec l'âge du gamétophyte, la taille étant la manifestation morphologique de l'âge, et que son unité d'expression est l'ovaire, les 10 ovules au sein d'une même capsule évoluant simultanément.

L'étude de l'influence de la température et de l'âge de la fleur permet d'appréhender 2 moyens d'augmenter le taux de polyembryonie. Si la castration suivie d'une pollinisation retardée permet effectivement d'accroître notablement le taux de polyembryons, ce dispositif est peu pratique à mettre en place et requiert un travail important. Par contre, l'influence favorable des hautes températures peut être facilement mise à profit pour obtenir un nombre élevé de polyembryons sans augmentation notoire du travail et des coûts. Les températures les plus favorables, $24^{\circ} \mathrm{C} / 18^{\circ} \mathrm{C} \mathrm{J} / \mathrm{N}$ et $28^{\circ} \mathrm{C} / 12^{\circ} \mathrm{C} \mathrm{J} / \mathrm{N}$, proches de celles observées par GREEN (com. pers.), $27^{\circ} \mathrm{C}$, permettent un taux moyen de 27,9 p. 100 et 26,8 p. 100 respectivement sur RA91 HD8 et constituent un moyen efficace pour la production d'un nombre élevé d'haploïdes issus de polyembryons.

\section{CONCLUSION}

L'utilisation des haploïdes et des haploïdes doublés permet d'accélérer les schémas de sélection des espèces allogames et autogames comme le lin. Chez cette espèce, la polyembryonie haploïde-diploïde, dont l'origine se situe dans la division de l'oosphère avant fécondation est une voie prometteuse de production de structures haploïdes. L'optimisation des conditions de culture permet une augmentation sensible des taux de production. Cependant, l'utilisation de ce phénomène nécessite la connaissance du déterminisme génétique et le transfert du caractère dans le matériel végétal en sélection.

Reçu le 17 novembre 1986. Accepté le 12 avril 1987. 


\section{RÉFÉRENCES BIBLIOGRAPHIQUES}

Blackburn D. T., Christophel D. C., 1976. A method of permanently mounting biological tissue cleared in Herr's four-and-a-half clearing fluid. Stain Technology, 51, 125-130.

Cooper D. C., 1943. Haploid-diploid twin embryos in Lilium and Nicotiana. Amer. J. Bot., 30, 408-413.

Dumas de Vaulx R., 1977. Embryogenèse des plantules haplö̈des chez le piment (Capsicum annuum L.). Proceedings 3rd Congress Eucarpia Capsicum Avignon-Montfavet ed. by I.N.R.A., 67-73.

Green A. G., Salisbury P. A., 1983. Inheritance of polyembryony in flax (Linum usitatissimum L.). Can. J. Genet. Cytol., 25, 117-121.

Herr J. M., 1971. A new clearing squash technique for the study of ovule developpment in Angiosperms. Amer. J. Bot., 58, 785-790.

Huyghe G., 1985. Etude physiologique et génétique de la polyembryonie haploide-diploïde chez le lin (Linum usitatissimum L.). Thèse de Docteur-Ingénieur, ENSA-Université Rennes 1.

Kappert H., 1933. Erbliche Polyembryonie bei Linum usitatissimum. Biol. Zentralbl. 53, 276-307.

Kennell J. C., Horner H. T., 1985. Influence of the soyabean male sterile gene (msl) on the development of the female gametophyte. Can. J. Genet. Cytol., 27, 200-209.

Leviel C., Huyghe C., 1985. Observations des gamétogenèses mâle et femelle, de la fécondation et de la formation d'embryons non zygotiques après éclaircissage des anthères et des sacs embryonnaires de Cichorium intybus L. et de Linum usitatissimum L. C. R. Acad. Sci. Paris 301, Sér. 3, 7, 373-378.

Murray B. E., 1980. Analysis of meiotic metaphase in haploids and hybrids of haploid $\times$ diploid flax. Can. J. Genet. Cytol., 22, 597606.

Murray B. E., 1985. Studies of haploid-diploid twins in flax (Linum usitatissimum L.). Can. J. Genet. Cytol., 27, 371-379.

Plessers A. G., 1965. The use of haploids in flax breeding. 35th Annual Flax Institute of the U.S.

Rajhathy T., 1976. Haploid flax revisited. Z. Pflanzenzuchtung, 76, 1-10.

Ramiah K., Parthasarathy N., Ramanujam S., 1935. Polyembryony in rice (Oryza sativa). Ind. J. Agr. Sci., 5, 2, 119-124.

Randall T. E., Rick C. M., 1945. A cytogenetic study of polyembryony in Asparagus officinalis L. Amer. J. Bot., 32, 560569.

Thompson T. E., 1977. Haploid breeding technique in flax. Crop Sci., 17, 757-760.

Zverzhanskaya L. S., Grishina E. V., 1980. Cytoembryological study of haploidy and polyembryony in restitution lines of corn. Cytol. Genet., 14, 4, 1-6. 